\title{
\begin{tabular}{l|l|l|l|l}
\hline M & R & S & Internet Journal of & Nitride Semiconductor Research \\
\hline
\end{tabular}
}

Volume 2, Article 8

\section{Surface Morphology and Structure of $\mathrm{GaN}_{\mathrm{x}} \mathrm{As}_{1-\mathrm{x}}$}

\author{
J.V. Thordson, O. Zsebök, U. Södervall, T.G. Andersson \\ University of Göteborg, Sweden \\ and \\ Chalmers University of Technology
}

This article was received on June 9, 1997 and accepted on July 2, 1997.

\begin{abstract}
$\mathrm{GaN}_{\mathrm{x}} \mathrm{As}_{1-\mathrm{x}}$-layers were grown by solid source molecular beam epitaxy using $\mathrm{N}_{2}$ and excitation by RF-plasma source. The average nitrogen concentration, $x$, determined by secondary ion mass spectrometry, ranges from isovalent nitrogen doping in GaAs up to GaN. X-ray diffraction revealed two peaks, close to the ones for $\mathrm{GaAs}(002)$ and the $\mathrm{GaN}(002)$ diffraction, respectively. The position of both peaks moved slightly as a function of nitrogen content. Scanning electron microscopy indicated a rough surface structure with improved smoothing for low and high nitrogen compositions. The rough surface is partly due to crystallite formation when mixing arsenic and nitrogen as a result of phase separation between the GaAs and GaN. In ternary films with very high nitrogen composition there were structural features with a different contrast. These were crystallites that arose at the interface and grew up to the surface.
\end{abstract}

\section{Introduction}

The $\mathrm{GaN}_{\mathrm{x}} \mathrm{As}_{1-\mathrm{x}}$ alloy has a large span in lattice constant, $4.50-5.65 \AA$, and band gap, $1.42-3.45 \mathrm{eV}$, for the zinc blende structure. Thus, this alloy system covers a large energy spectrum of interest for emission and detection of visible light. One major problem for the group III-nitrides is the lack of suitable substrate material. It is interesting to note that zinc blende $\mathrm{GaN}_{\mathrm{x}} \mathrm{As}_{1-\mathrm{x}}$ is lattice matched to $\mathrm{Si}$ for $\mathrm{x} \approx 0.2$. However, it has been questioned if this alloy can be used. Instead of a near-linear dependence of the band gap between GaAs and $\mathrm{GaN}$, there is a much larger alloy band gap bowing than for other ternary semiconductors. The alloy band gap is strongly reduced from the values given by the binaries [1] [2] [3] [4] and the band gap is even below zero for the approximate range, $0.1<x<\sim 0.9$, giving a semimetal [1]. Using first principles supercell calculations the large band gap bowing is as a result of charge exchange and atomic size differences [2].

Scanning electron microscopy (SEM) studies [5] [6] of $\mathrm{GaN}_{x} A s_{1-x}$ revealed a surface which is very rough, opposite to the smoother pure GaN. Further, the X-ray diffraction (XRD) peak was found to be clearly broadened. Transmission electron microscopy (TEM) unveiled a columnar grain structure of a large number of small $\mathrm{GaN}$ crystallites [6]. It has been shown that $\mathrm{GaN}_{\mathrm{x}} \mathrm{As}_{1-\mathrm{x}}$ grown on $\mathrm{GaAs}$ gives rise to cubic material, while growth on GaP provides the hexagonal structure [6] [7]. Photoluminescence (PL) investigations for $x<0.1$ indicated an almost linear reduction in the band-to-band transition energy with nitrogen composition [3] [4] [8] [9]. Further, PL investigations have been made on ion implanted N in GaAs [10] [11], where excitonic emission of nitrogen pairs was detected, and atomic layer N-doped GaAs [12] in which excitons bound to $\mathrm{N}$ traps were the most intense ones. Kondow et al. [1] have succeeded to grow $2 \mathrm{~nm}$ thick $\mathrm{GaN}_{\mathrm{x}} \mathrm{As}_{1-\mathrm{x}} / \mathrm{GaP}$ strained quantum wells on a GaP substrate with $10 \%$ nitrogen. In this case the photoluminescence emission from the well provided a large band gap at $2.044 \mathrm{eV}$.

In this work we report studies of growth and characterisation of $\mathrm{GaN}_{\mathrm{x}} \mathrm{As}_{1-\mathrm{x}}$ layers on $\mathrm{GaAs}(001)$ substrates where the nitrogen content was increased from the isovalent doping range, up to GaN. In order to control the 
nitrogen composition during growth the nitrogen- and arsenic fluxes were varied. The average nitrogen composition, $\mathrm{x}_{\mathrm{avg}}$, was determined by secondary ion mass spectrometry (SIMS). Characterisation by high resolution, double crystal XRD gave rise to two features, one close to the $\mathrm{GaAs}(002)$ peak and the other one at the $\mathrm{GaN}(002)$ peak. Both the peak positions and their intensities are characteristic of the alloy and changed slightly as a function of the average $\mathrm{N}$-content. The GaN related peak disappeared at low $\mathrm{x}_{\mathrm{avg}}$ and the one close to $\mathrm{GaAs}$ was absent for $\mathrm{x}_{\mathrm{avg}}>0.95$. At higher nitrogen compositions, SEM studies revealed a smooth surface but with observable crystalline features. The general surface roughness increased with mixing between As and N, and layers in the middle of the compositional range gave the worst crystal quality. The results are discussed in terms of phase separation. The paper is organised in the following way: After describing the experimental proceedings, we present results and discuss the nitrogen concentration in the samples and its change with growth parameters. Finally, the XRD and SEM results are analysed and discussed.

\section{Experimental}

The layers were grown with a solid source Varian GEN II Modular system on In-free mounted semiinsulating GaAs substrates. The arsenic flux was controlled by a valved cracker. The active nitrogen was provided by a liquid nitrogen cooled Oxford Applied Research CARS25 RF activated plasma source, capable of running at a power up to $600 \mathrm{~W}$. The nitrogen flow was controlled by a flow meter supplied from a gas tank filled from boiling liquid nitrogen. Pumping of the growth chamber was made by a $1500 \mathrm{l} / \mathrm{s}$ cryo pump and a $1500 \mathrm{l} / \mathrm{s}$ magnetic-bearing turbomolecular pump.

We divide the structures into three categories depending on the average nitrogen content in the layers. These are: GaAs:N-layers containing low concentrations of isovalent doping nitrogen, As-rich $\mathrm{GaN}_{\mathrm{x}} A s_{1-\mathrm{x}}$, with $\mathrm{x}$ from 0.01 , up to 0.3 , and finally As-poor $\mathrm{GaN}_{\mathrm{x}} A s_{1-\mathrm{x}}$-layers, with nitrogen concentrations of $\mathrm{x}_{\mathrm{avg}}>0.95$. For growth of layers with $x_{a v g} \leq 0.3$, the substrate temperature was kept at $580^{\circ} \mathrm{C}$ throughout the structure. A $1.00 \mathrm{sccm} \mathrm{N}$ $2^{-f l u x}$ was introduced with the $\mathrm{N}$-shutter closed. After this the power of the plasma source was increased in several steps up to $600 \mathrm{~W}$ to avoid overheating. During this start-up-procedure of the nitrogen source special precausions were taken to avoid nitridation of the surface. Therefore the nitrogen shutter was opened after the GaAs-growth was initiated. During the isovalent doping procedure the growth took place in a nitrogen atmosphere of $\sim 10^{-6}$ torr and the plasma was not ignited (but the RF-power was on). In this case average doping concentrations in the $10^{18}-10^{19} \mathrm{~cm}^{-3}$ range were measured by SIMS. If the source was operated in the normal mode, i. e. the plasma was switched on after ramping up the power to $600 \mathrm{~W}$, an average nitrogen composition representing a ternary alloy was obtained. For the growth of As-poor layers $\mathrm{GaN}_{\mathrm{x}} A \mathrm{~s}_{1-\mathrm{x}}$ with high $\mathrm{x}$-values, above 0.95 , a slightly different procedure was used. The growth temperature was $600^{\circ} \mathrm{C}$ during these layer depositions. First, GaAs deposition at $0.3 \mu \mathrm{m} / \mathrm{h}$ was established. Thereafter, the RF power was increased to $600 \mathrm{~W}$ and the plasma ignited, maintaining a $0.6 \mathrm{sccm}$ nitrogen flux. When growing the buffer layer, both $\mathrm{As}$ and $\mathrm{N}$ were supplied during 2 to 4 minutes, until the reconstruction in the reflection high energy diffraction (RHEED)-pattern disappeared (giving 1x1 structure). When the buffer layer deposition was finished, the arsenic needle valve was changed from $1 \times 10^{-5}$ torr to the preferred As beam flux pressure for the $\mathrm{GaN}_{\mathrm{x}} \mathrm{As}$ 1 - $x^{-l a y e r}$ growth, which was in the range of $2 \times 10^{-7}-2 \times 10^{-6}$ Torr. In the case of pure GaN-growth, both As-shutter and As-cracker valve were closed. At the same time the Ga furnace temperature was decreased to reduce the growth rate from 0.3 to $0.2 \mu \mathrm{m} / \mathrm{h}$ (with reference to GaAs-growth). The majority of the samples were $4 \mu \mathrm{m}$ thick, except for the As-poor layers with $\mathrm{x}>0.95$, which were approximately $1 \mu \mathrm{m}$ thick.

The determination of the average composition was carried out by SIMS, using $\mathrm{Cs}^{+}$as primary ions. The intensities of $\mathrm{O}^{-}, \mathrm{Ga}^{-}, \mathrm{GaN}^{-}, \mathrm{As}^{-}$and $\mathrm{C}^{-}$signals were recorded within. The single crystalline structure was characterised by double crystal XRD. High resolution secondary electron (SE) images were taken by scanning electron microscope (SEM) operating with $25 \mathrm{keV}$ electron accelerating voltage. For cross sectional SEM investigations the $\{110\}$ cleavage planes of the GaAs substrate were used. To measure the relative GaN content in features with different surface contrast, energy dispersive X-ray spectrometry (EDX) was made.

\section{Results and discussion}

Measurements by XRD and SIMS were performed to determine the nitrogen content of the samples. A typical SIMS in-depth profile from a $4 \mu \mathrm{m}$ thick $\mathrm{GaN}_{\mathrm{x}} A s_{1-\mathrm{x}}$ layer, $\mathrm{x}_{\mathrm{avg}}=0.18$, is shown in Figure 1 . The intensity for

the mass $83\left(\mathrm{GaN}^{-}\right)$was used since the sensitivity for both $\mathrm{N}^{+}$and $\mathrm{N}^{-}$-ions is very low. The growth process produced some unintentional impurities, mostly carbon and oxygen. As shown the carbon signal has a maximum at the film/substrate interface and at the surface of the layer, both exposed to the atmosphere. The 
SIMS measurements also reveal the presence of some oxygen in the layers. The content of this increases with nitrogen composition (as compared to a GaAs film) due to outgassing in the nitrogen plasma source.

There are two principal problems in governing the nitrogen content. The first is to control the column $\mathrm{V}$ flux, especially nitrogen, and secondly to measure a correct composition, particularly if the sample is not homogeneous. For low arsenic concentration in GaN the arsenic beam flux pressure was taken as a measure while the optical emission signal from the plasma source was the growth reference for low nitrogen alloying at GaAs growth. To determine the alloy content by SIMS the signals were normalised to Ga providing the signal ratios $\mathrm{As} / \mathrm{Ga}$ and $\mathrm{GaN} / \mathrm{Ga}$, respectively. In alloy compositions the relative sensitivity of SIMS for the different masses continuously vary between $\mathrm{GaAs}$ and $\mathrm{GaN}$ due to the change of the matrix. Since there is no information available about this we have assumed the sensitivity to be fairly constant. The GaN/Ga-ratio was used to assess the $\mathrm{N}$-concentration in the samples with $\mathrm{x}<0.3$. The isovalently $\mathrm{N}$-doped layers were calibrated against a sample with ion implanted nitrogen in GaAs. This might be useful for compositions up to the solubility limit (1-2\%). To determine the absolute values, the GaN/Ga-ratio was normalised to the ratio for GaN, which equals $\sim 75$. For high nitrogen concentrations $\left(x_{a v g}>0.95\right)$ the As/Ga-ratio is a more sensitive parameter to determine the composition. In this case the As/Ga-ratio was normalised to one of the nitrogen doped samples. Figure 2 summarises the measured data where the As/Ga-ratio is shown as a function of GaN/Ga-ratio as provided by SIMS where the measured data are expected to fall within the marked area. The reason for such an uncertainty is that different results are obtained between two measurements on same sample or two samples grown under identical conditions which demonstrates the statistical spread of these ratios. We expect the SIMS data to be within a factor of 2 for $x<1 \%$ and $x>99 \%$, and then a rather large variation in remaining $\mathrm{N}$-compositional range.

In the growth procedure of samples with 1 to $30 \%$ nitrogen we referred the $\mathrm{N}$-concentration to the signal intensity from the nitrogen plasma optical emission which is expected to be a measure of the cracking efficiency, and therefore the amount of nitrogen content. In order to minimise the number of parameters, we kept the RF-power constant at $600 \mathrm{~W}$, and varied the $\mathrm{N}$-flux between 0.05 and $1.00 \mathrm{sccm}$. In this case we could obtain $x_{a v g}>5 \%$ but with increasing variation in $x_{a v g}$ with nitrogen content. The reason for this might be inhomogeneities in the plasma and in the sample, partly due to the large mismatch between substrate and film, seen in Figure 3. In order to reach a lower nitrogen composition (but higher than doping concentration) both the RF-power and nitrogen flux must be reduced. A value of $x_{a v g} \approx 1.6 \%$ was obtained for $300 \mathrm{~W}$ and a $\mathrm{N}$ $2^{\text {flow of }} 0.1 \mathrm{sccm}$. For high nitrogen concentrations, the GaN growth was established and the As-pressures were varied by the needle valve, the upper part of Figure 3. There is a systematic increase in As-content with the flux and here the compositional variations are much lower than in the other end of the alloy range.

In Figure 4 the XRD spectrum from a layer with an average-composition of $\sim 20 \%$ is shown. There are two evident features, one peak is very close to the GaAs peak and there is another rather broad peak at $\sim 43^{\circ}$, which is asserted to the (002) zinc blende GaN. The two extra peaks are highlighted in the insets. Assuming the $\mathrm{GaAs}$-derived peak is from $\mathrm{Ga}_{x} \mathrm{~N}_{1-x} \mathrm{As}$ (with a low concentration of nitrogen) and applying the linear Bragg

and Vegard's law on it, the N-composition will be about one order of magnitude smaller as from the SIMS measurements. The SIMS-composition is not expected to be uncertain within one order of magnitude, so at least a part of the difference is explained by the formation of $\mathrm{GaN}(\mathrm{As})$. In the inset is indicated that the GaAs-like signal is quite intense at low nitrogen compositions but with increasing $x_{\text {avg-values }}$ it becomes fainter, and at compositions close or equal to 1 they are extinct. The opposite trends are illustrated in the other inset, showing the GaN-feature. Consequently, for samples with the lowest nitrogen content there is no GaN-diffraction but the GaN arise when nitrogen is above the solubility level in GaAs. Another interesting remark is that the peak maximum is shifted from the GaN-position with increasing arsenic concentration. In Figure 5 is seen that the position of both the GaAs and the (002) GaN derived XRD-peaks depends on the nitrogen content. This is partly due to the strain forces induced into the two materials and partly that some nitrogen and arsenic is solved into the binaries. At high nitrogen concentrations the intensity of the GaAs-related peak has been reduced. As stated above the discrepancy of the N-content between SIMS and $\mathrm{XRD}$, is an effect of a phase separation, one consisting of GaAs with solved N, and the other of GaN with As. It was also seen that the relative GaN peak area increased with nitrogen content, and with a steep change close to pure GaN, at the same time as its FWHM decreases. Evidently the GaN quality is rapidly reduced with a small amount of solved arsenic.

In the characterisation of surfaces by SEM we found that the isovalently N-doped GaAs samples had same surface morphology as GaAs. Samples with high (95-100\%) or low (1 -20\%) average nitrogen alloy-concentration had different morphologies. In the lower nitrogen composition range, a homogeneous distribution of elliptically shaped openings (holes) with a diameter of 3-4 $\mu \mathrm{m}$ were observed. The surface area between the holes was quite smooth. The holes were seen to grow with a conical shape as a function of the layer thickness. The tip of the cone was in the layer and the void was possibly formed at the position of 
dislocations arising due to lattice mismatch. Similarly shaped holes have previously been observed by SEM after dislocation etching on surfaces of GaN grown on sapphire [13]. The samples with compositions, between 20 and $30 \%$, (with the highest degree of mixing) gave the worst surface morphology with typically $1 \mu \mathrm{m}$ surface roughness due to crystallite formation at Figure 6 . This is interpreted as crystallites separated into two phases. With increasing $\mathrm{N}$-content, $\mathrm{x}_{\mathrm{avg}}>0.95$, the surface became increasingly smooth. For the sample with $\sim 4 \%$ As, the surface roughness is smaller $(<1 \mu \mathrm{m})$ with less crystallite structure and in samples with $\sim 0.5 \%$ As, Figure 7, the surface smoothness has improved to below $\sim 100 \mathrm{~nm}$. Two types of features were seen, one having a cylindrical shape, $1 \times 3 \mu \mathrm{m}$, not very different compared to the $4 \%$ As sample. Analysis by EDX revealed an increased GaN composition is these as compared to the rest of the surface, judged from the $\mathrm{N}$-peak intensity despite that EDX analysis has a rather large interaction region. The other type of feature has a rather spherical shape, $\sim 1 \mu \mathrm{m}$ in diameter. Analysis by EDX of this indicated a slightly lower GaN concentration compared to the rest of the surface.

\section{Conclusion}

Thin epitaxial layers of $\mathrm{GaN}_{\mathrm{x}} \mathrm{As}_{1-\mathrm{x}}$ were grown on $\mathrm{GaAs}(001)$ substrates by solid source MBE and an RF activated plasma source. The average nitrogen composition has been varied from isovalent doping concentration, $10^{18}-10^{19} \mathrm{~cm}^{-2}$, up to GaN. By growing GaAs in a nitrogen atmosphere with the nitrogen plasma extinct the isovalent $\mathrm{N}$-doping concentrations in $\mathrm{GaAs}$ were obtained. The $\mathrm{N}$-content was controlled by varying the $\mathrm{Ga}$ growth rates, the arsenic and nitrogen fluxes and the RF-power. The average nitrogen composition was determined by SIMS. The structural composition given by XRD indicated two different diffraction peaks, one close to the $\mathrm{GaAs}(002)$ and one close to the $\mathrm{GaN}(002)$ position. The position of both peaks slightly moved towards the high angle side when increasing the nitrogen concentration. Considering the peak beside the $\mathrm{GaAs}(002)$ peak arose from the $\mathrm{GaN}_{\mathrm{x}} A s_{1-\mathrm{x}}$ and use Bragg and Vegard's laws on it, there is a discrepancy in about one order of magnitude between the XRD and SIMS composition. SEM indicates holeslayers having a few percent nitrogen composition samples which are formed due to the lattice mismatch to the substrate. At high nitrogen concentrations, features on the surface with a difference in contrast are seen which are crystallites. The two XRD-peaks close to GaAs and GaN, respectively, give evidence to a phase separation between the two materials, where the crystallites seen in the SEM micrographs consist of a higher GaN-composition, shown by EDX.

\section{Acknowledgments}

We acknowledge A. Kvist for his assistance in the EDX analysis.

\section{References}

[1] Shiro Sakai, Yoshihiro Ueta, Yoji Terauchi, Jpn. J. Appl. Phys. 32, 4413 (1993).

[2] Su-Huai Wei, Alex Zunger, Phys. Rev. Lett. 76, 664-667 (1996).

[3] Markus Weyers, Michio Sato , Appl. Phys. Lett. 62, 1396-1398 (1993).

[4] Markus Weyers, Michio Sato, Hiroaki Ando, Jpn. J. Appl. Phys. 31, L853 (1992).

[5] L. C. Jenkins, T. S. Cheng, C. T. Foxon, S. E. Hooper, J. W. Orton , S. V. Novikov, V. V. Tret'yakov , J. Vac. Sci. Technol. B 13, 1585-1590 (1995).

[6] Y. Xin, P. D. Brown, R. E. Dunin-Borkowski, C. J. Humphreys, T. S. Cheng, C. T. Foxon, J. Cryst. Growth 171, 321 (1997).

[7] S. Bharatan, K. S. Jones, C. R. Abernathy, S. J. Pearton , F. Ren, P. W. Wisk, J. R. Lothian , J. Vac. Sci. Technol. A 12, 1094-1098 (1994).

[8] Masahiko Kondow, Kazuhisa Uomi, Kazuhiko Hosomi, Teruo Mozume, Jpn. J. Appl. Phys. 33, L1056 (1994).

[9] Michio Sato, J. Cryst. Growth 145, 99 (1994). 
[10] I. P. Akimchenko, N. V. Dymova, V. V. Chaldyshev, Yu. V. Shmartsev, Sov. Phys. Semicond. 24, 1155 (1990).

[11] Takayuki Shima, Yunosuke Makita, Shinji Kimura, Tsutomu lida, Xiaohua Fang, De-sheng Jiang, Kazuhiro Kudo, Kuniaki Tanaka, Nucl. Instr. Meth. Phys. Res. B 120, 293 (1996).

[12] Toshiki Makimoto, Naoki Kobayashi, Appl. Phys. Lett. 67, 688 (1995).

[13] Takahiro Kozawa, Tetsu Kachi, Takeshi Ohwaki, Yasunori Taga, Norikatsu Koide, Masayoshi Koike, J. Electrochem. Soc. 143, L17 (1996).

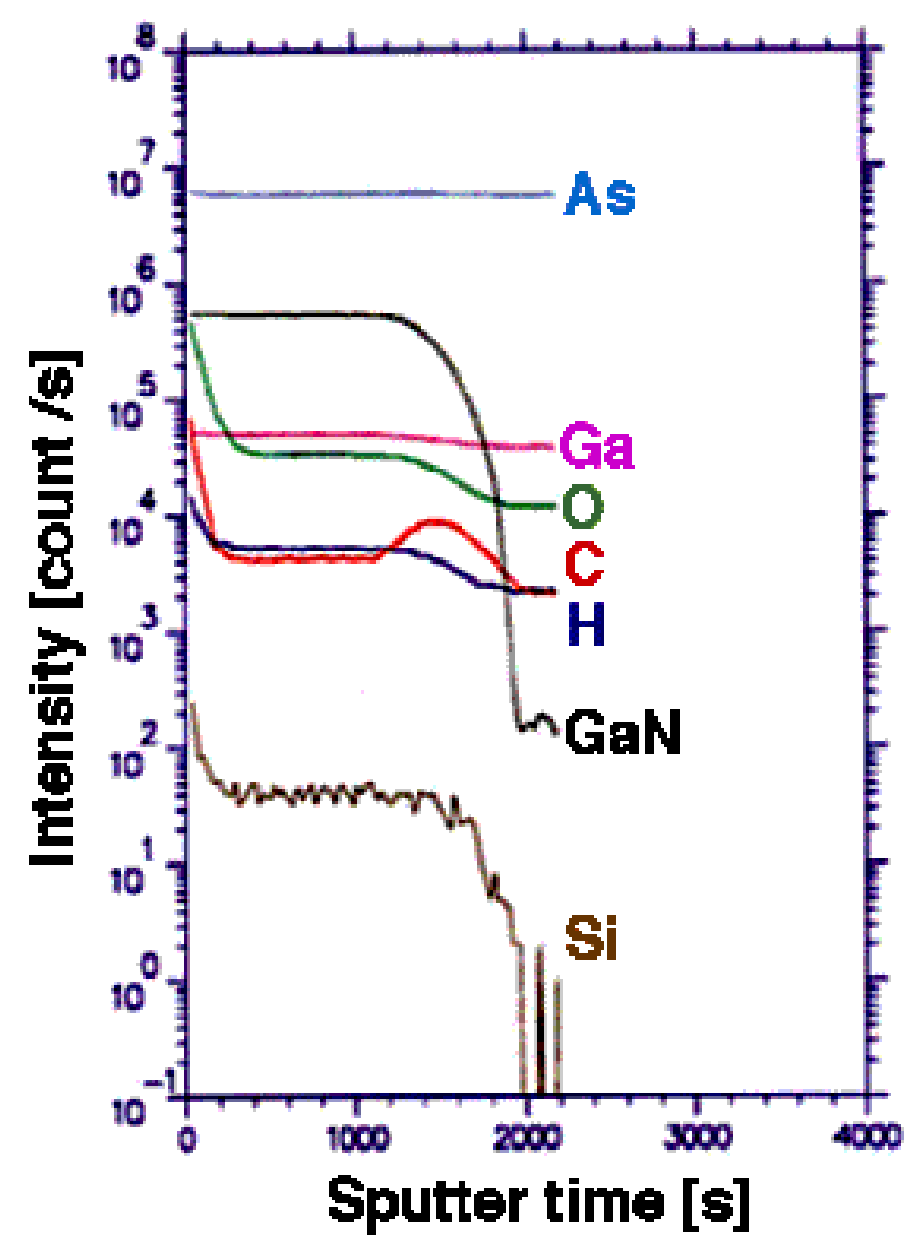

Figure 1. SIMS profile of a $4 \mu \mathrm{m}$ thick $\mathrm{GaN}_{0.2} \mathrm{As}$ 0.8 as a function of the sputtering time. The interface to the GaAs substrate is clearly seen. 


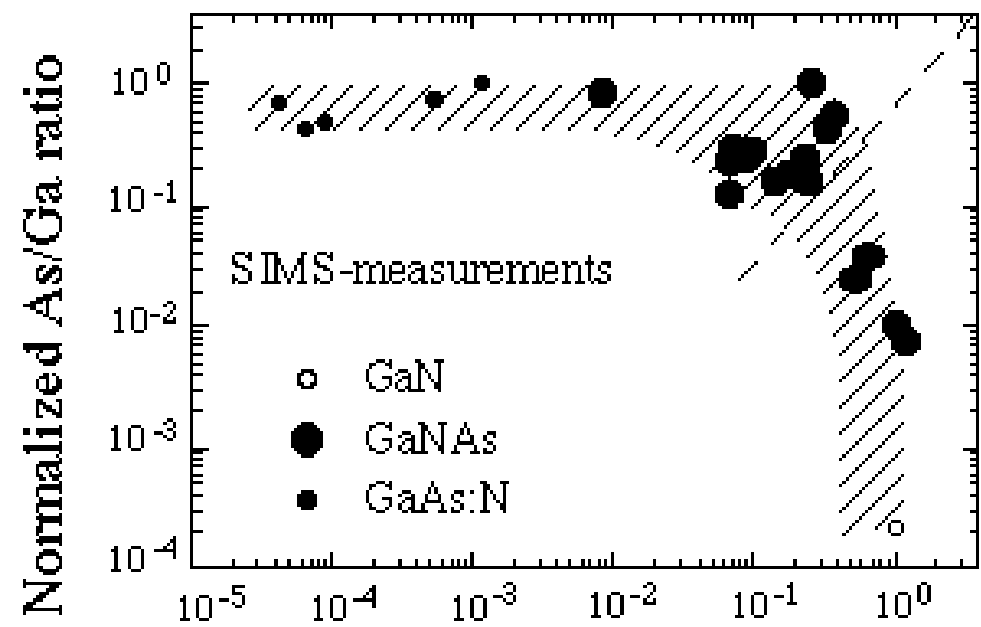

Normalized $\mathrm{GaN} / \mathrm{Ga}$ ratio
Figure 2. The As/Ga-ratio as a function of the GaN/Ga-ratio from the SIMS-data. The N-doped GaAs-samples and the $\mathrm{GaN}_{\mathrm{x}} \mathrm{As}_{1-\mathrm{x}}, \mathrm{x}<0.3$ were calibrated with reference to the GaN/Ga-ratio, and the high nitrogen composition samples were calibrated to the As/Ga-ratio. The SIMS analysis gives the average nitrogen composition in the samples.

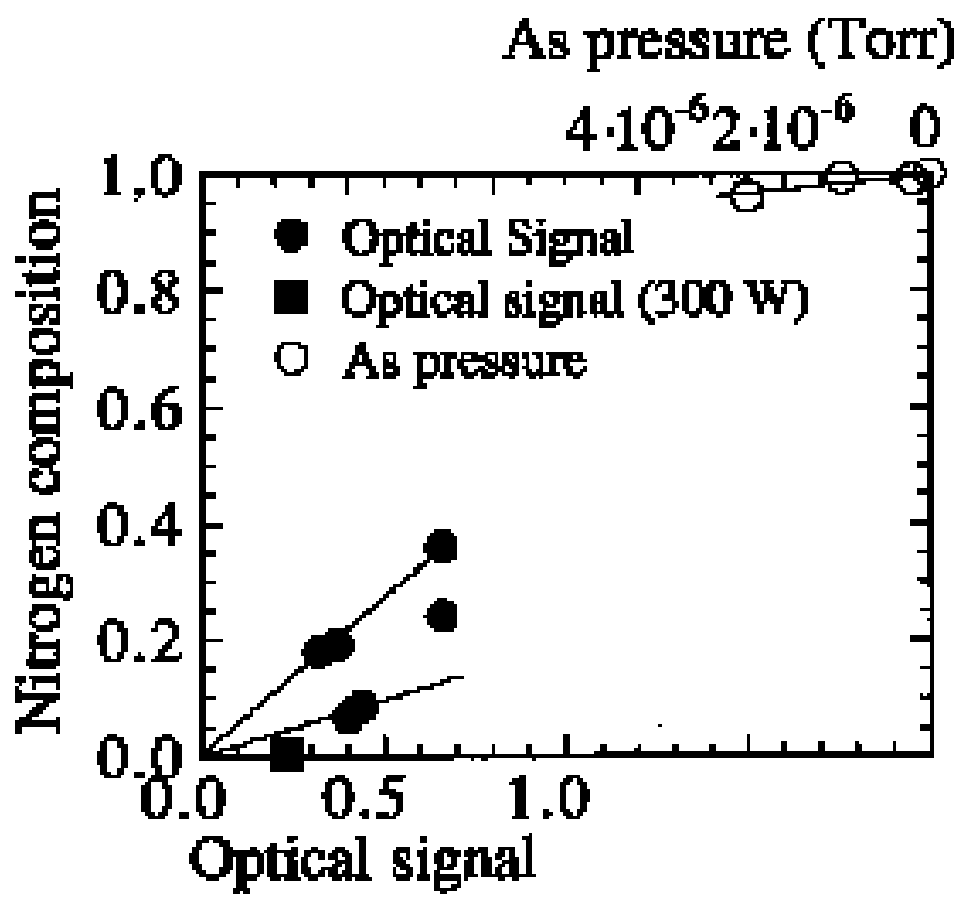

Figure 3. The average nitrogen composition as a function of growth parameters. The nitrogen concentration in $\mathrm{GaAs}(\mathrm{N})$ was controlled by the light intensity from the plasma (optical signal) and the arsenic concentration in $\mathrm{GaN}(\mathrm{As})$ was controlled by the arsenic beam pressure. There is a substantial spread in nitrogen content, even when using identical growth parameters, $600 \mathrm{~W}$ and $1 \mathrm{sccm}$ of $\mathrm{N}_{2}$. For the lowest average nitrogen concentration, $1.6 \%$, only $300 \mathrm{~W}$ RF power was used and a nitrogen flux of 0.1 sccm.

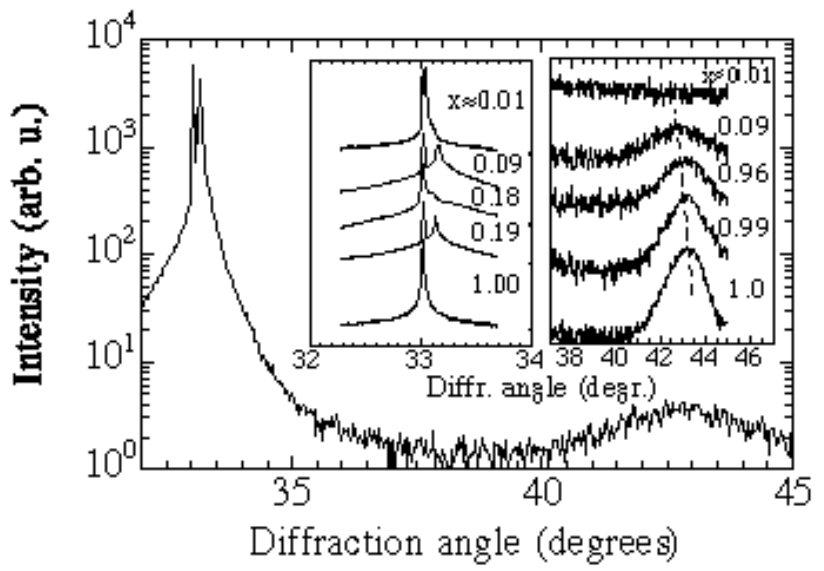

Figure 4. The XRD spectrum for a $\mathrm{GaN}_{0.2} \mathrm{As}_{0.8}$ sample where both a GaAs(002)- and a $\mathrm{GaN}(002)$ peak from the layer can be seen. The insets show a shift of the peaks and that the GaAs-layer peak disappears at high $x$-compositions, while the opposite happens to the GaN feature. 


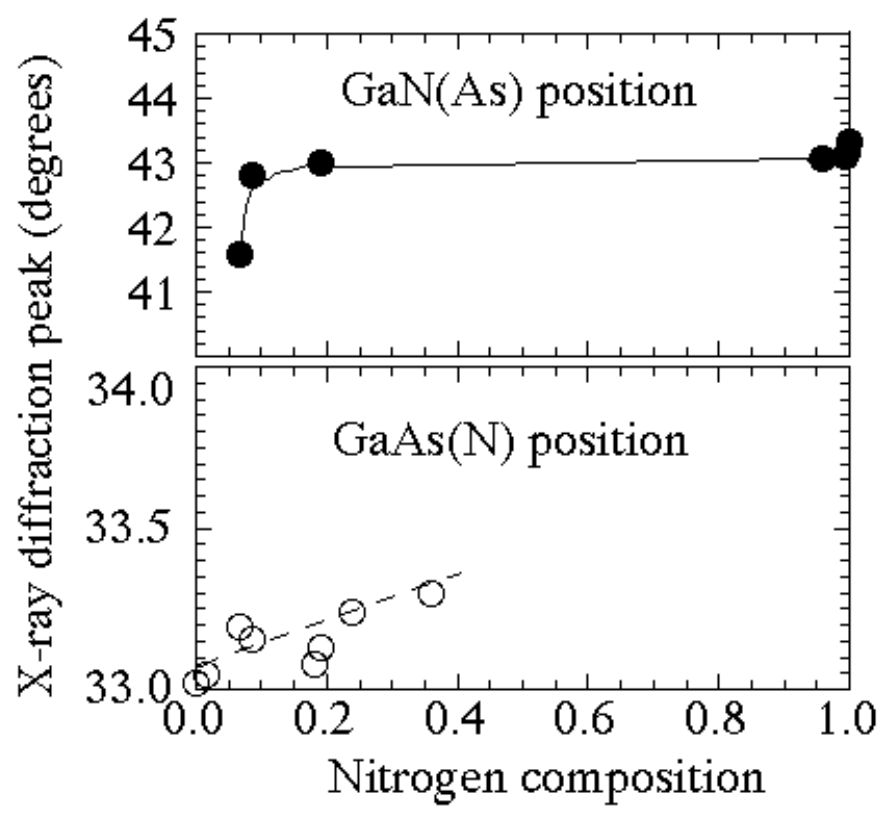

Figure 5. The positions of the $\mathrm{GaAs}(\mathrm{N})$ - and $\mathrm{GaN}(\mathrm{As})$ peaks as a function of the average nitrogen composition. Both features move towards the high-angle side with nitrogen content.

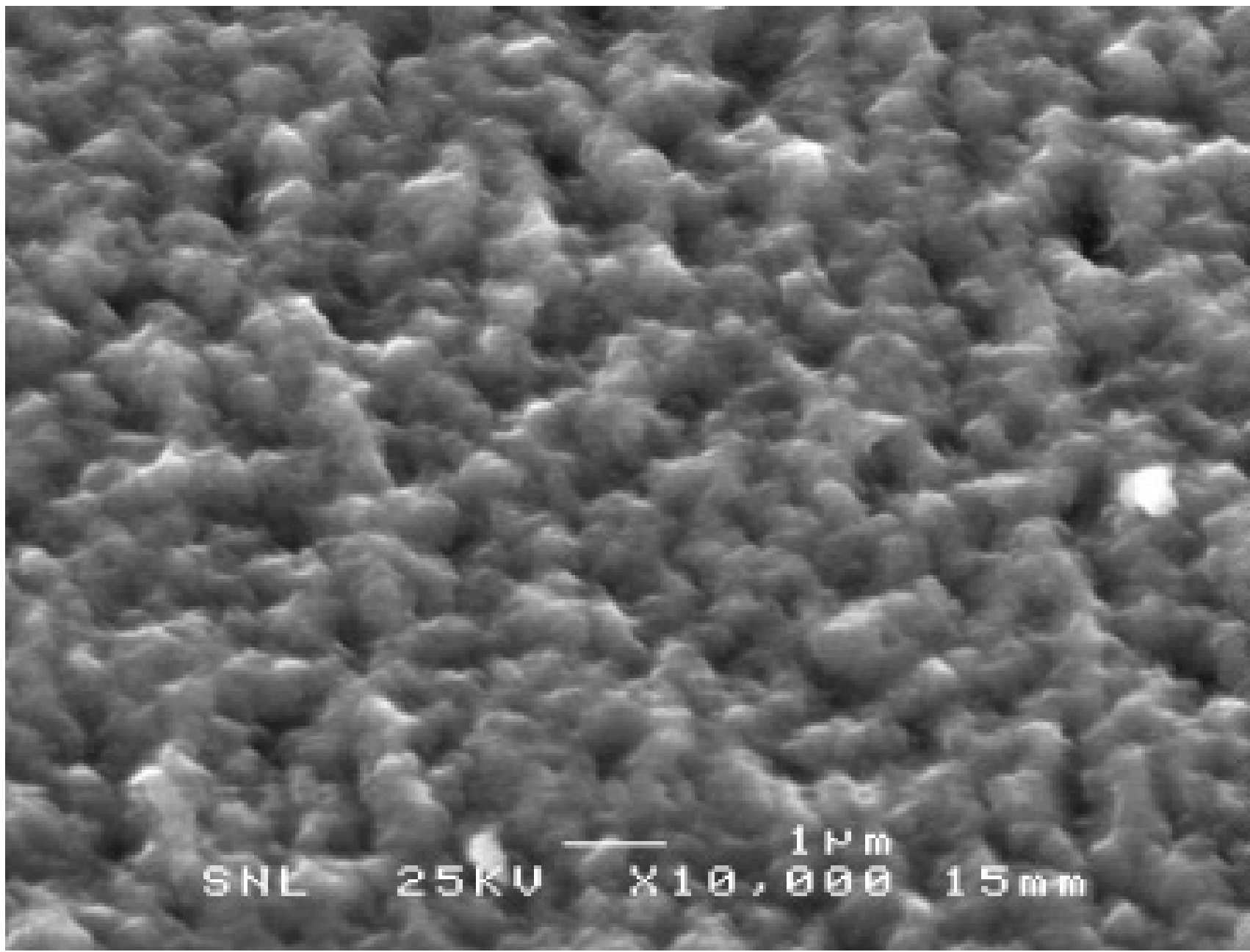

Figure 6. SEM-micrograph showing the morphology of $\mathrm{GaN}_{\mathrm{x}} A s_{1-x}$ where the average nitrogen composition is $\sim 30 \%$. The surface roughness is due to crystallites. 


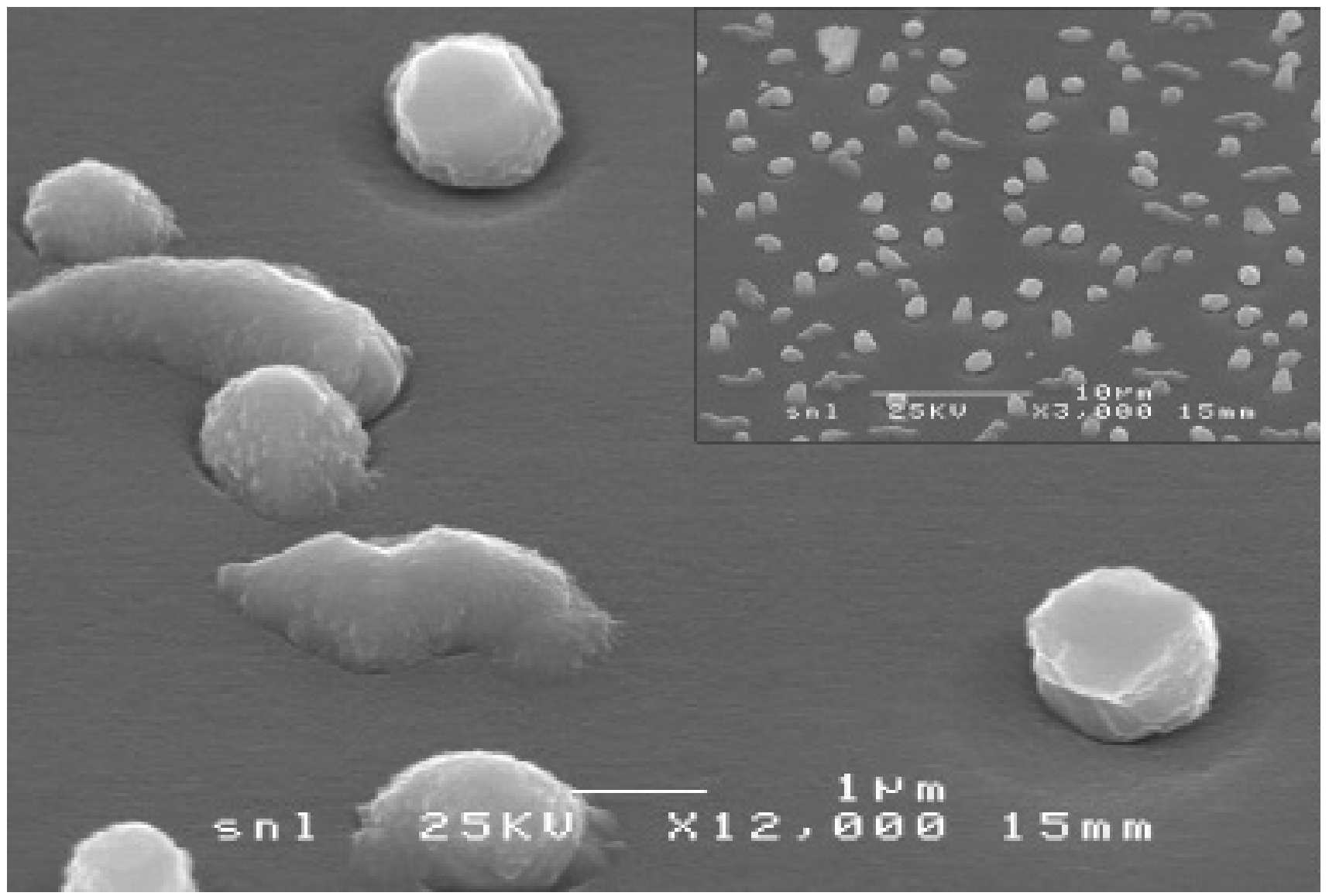

Figure 7. SEM-micrograph of a GaN(As) surface with $0.5 \%$ As. The approximately $1 \mu \mathrm{m}$ sized round and prolonged features are discussed in the text.

(C) 1997 The Materials Research Society

\begin{tabular}{|c|c|c|c|c|}
\hline $\mathrm{M}$ & $\mathrm{R}$ & $\mathrm{S}$ & Internet Journal of & Nitride Semiconductor Research \\
\hline
\end{tabular}

